\title{
Tumor neuroectodérmico de seios paranasais: diagnóstico e tratamento
}

\author{
Primitive neuroectodermal tumor of paranasal sinuses: \\ diagnosis and treatment
}

\author{
Francisco das Chagas Cabral-Júnior, Fábio de Rezende Pinna², \\ Richard Louis Voegels ${ }^{3}$
}

Cabral-Júnior FC, Pinna FR, Voegels RL. Tumor neuroectodérmico de seios paranasais: diagnóstico e tratamento / Primitive neuroectodermal tumor of paranasal sinuses: diagnosis and treatment. Rev Med (São Paulo). 2012;91(3):219-22.

\begin{abstract}
RESUMO: Tumores neuroectodérmicos primitivos (PNET) são neoplasias raras e extremamente agressivas encontradas principalmente em crianças e adultos jovens. São classificados em periféricos ou centrais, de acordo com sua origem. O diagnóstico baseia-se na história clínica, sendo essenciais exames de imagem, como tomografia computadorizada e ressonância magnética, e, para a confirmação, estudo anatomopatológico. É importante distingui-los de outros tumores de células redondas pequenas, como linfoma, sarcoma de Ewing extraósseo e rabdomiossarcoma, exigindo diferenciação imunoistoquímica através de marcadores específicos. O tratamento envolve cirurgia, quimioterapia e radioterapia, sendo o prognóstico pobre e a sobrevida bastante reservada. O objetivo deste artigo é discutir as características clínicas, radiográficas e histológicas dos tumores neuroectodérmicos primitivos e seu tratamento.
\end{abstract}

DESCRITORES: Tumores neuroectodérmicos primitivos periféricos/patologia; Sarcoma de Ewing/patologia; Seios paranasais

\begin{abstract}
Primitive neuroectodermal tumours (PNET) are rare and highly aggressive neoplasms found mainly in children and young adults. They are classified in peripheral or central according to their origin. The diagnosis is based on clinical history, computed tomography and magnetic resonance imaging, but the pathological study is the only way to confirm it. It's very important to distinguish the PNET from other small cell round cell tumors - such as lymphoma, Ewing's sarcoma and rhabdomyosarcoma - through immunohistochemical specific markers. The treatment includes surgical resection, chemotherapy and irradiation. The prognosis is poor and the survival rate is variable. This article aims to discuss the clinical, imaging and histological features of the primitive neuroectodermal tumors and their treatment.
\end{abstract}

KEYWORDS: Neuroectodermal tumors, primitive, peripheral; Ewing's sarcoma/pathology; Paranasal sinuses

\footnotetext{
${ }^{1}$ Médico Residente de Otorrinolaringologia da Faculdade de Medicina da Universidade de São Paulo.

${ }^{2}$ Médico Assistente, Chefe do Grupo de Rinologia do HCFMUSP.

${ }^{3}$ Professor Livre-docente da Faculdade de Medicina da Universidade de São Paulo.

Endereço para correspondência: Av. Dr. Enéas Carvalho de Aguiar, 255. $6^{\circ}$ andar. Sala 6167. São Paulo, SP. CEP: 05403-900.
} 


\section{INTRODUÇÃO}

Os tumores neuroectodérmicos primitivos (PNETs) são neoplasias extremamente raras que geralmente acometem crianças e adolescentes ${ }^{1,2}$. Atualmente são divididos em centrais (CPNET) ou periféricos ( $\mathrm{pPNET}$ ), de acordo com sua origem no sistema nervoso central ou fora do cérebro e do sistema nervoso ${ }^{3}$. Estima-se que eles representem uma incidência de $4 \%$ dos tumores de partes moles ${ }^{4}$. Sua diferenciação com outros tumores de células redondas pequenas - como sarcoma de Ewing, sarcoma de Ewing extraósseo (extraesquelético), rabdomiossarcoma embrionário, neuroblastoma e linfoma - pode ser muito difícil ${ }^{5}$.

Eles surgem principalmente no sistema nervoso central na forma de meduloblastoma ${ }^{6}$, enquanto as formas periféricas são mais raras e podem crescer na parede torácica (tumor de Askin) ${ }^{4}$, região paravertebral ${ }^{4}$, pâncreas ${ }^{7}$, rins $^{8}$, útero ${ }^{9}$, vulva ${ }^{10}$ ou seios paranasais ${ }^{11}$. Dentre os tumores periféricos, a região mais acometida é o tórax (46\%) ${ }^{11}$. A região da cabeça e pescoço pode ser a origem do tumor em $24 \%^{12}$ a $42 \%$ dos pacientes ${ }^{11}$. O objetivo deste artigo é descrever a apresentação clínica e o diagnóstico desta rara entidade clínica, bem como discutir o tratamento preconizado e seu prognóstico.

\section{REVISÃO DA LITERATURA}

Os pPNET são classificados como sendo parte da família dos tumores de Ewing ${ }^{13}$, juntamente com o sarcoma de Ewing, neuroepitelioma, sarcoma de Ewing atípico e tumor de Askin (tumor da parece torácica), pois apresentam características semelhantes. Assim como o sarcoma de Ewing, os PNETs apresentam células pequenas, arredondas e hipercromáticas, pouco diferenciadas, sendo a distinção entre ambos feita pela presença de diferenciação neural (ultraestrutural ou imunoistoquímica) encontrada nos PNETs ${ }^{14}$. Dessa forma, o sarcoma de Ewing e o pPNET representam duas entidades de um mesmo espectro de tumores com vários graus de diferenciação histológica.

Sugere-se que os tumores neuroectodérmicos primitivos tenham origem na crista neural. Durante o desenvolvimento embrionário precoce, as células da crista neural migram dorsal e lateralmente, participando da organogênese do sistema nervoso periférico e endócrino ${ }^{15}$. Uma alteração genética, levando a translocação entre os braços longos dos cromossomos 11 e $22 \mathrm{t}(11 ; 22)$ (q24;q12), codifica uma proteína que provoca uma transformação maligna das células da crista neural com tendência a proliferação e invasão dos tecidos adjacentes ${ }^{1}$. Esta alteração também é observada nos sarcomas de Ewing ${ }^{1}$, bem como a expressão do produto gênico MIC-2 (CD99), localizado no braço curto do cromossomo 6 (não encontrada nos cPNETs). Como citado anteriormente, os pPNETs e os sarcomas de Ewing apresentam características histológicas semelhantes com graus distintos de diferenciação, tendo os pPNETs mais propriedades dos tumores neurogênicos, incluindo positividade para marcadores neuronais (enolase neurônio-específica - NSE - e sinaptofisina) ${ }^{16}$ e para vimentina e cromogranina ${ }^{11}$.

Os tumores neuroectodérmicos, de uma forma geral, são discretamente mais comuns em homens e jovens ( $<25$ anos) e os periféricos costumam ser mais invasivos e metastáticos que os centrais ${ }^{16}$. A sintomatologia varia de acordo com a localização do tumor. Os pacientes usualmente abrem o quadro com uma massa dolorosa e sintomas constitucionais, alcançando tamanhos extensos no momento do diagnóstico (até 20 centímetros) $)^{1,5}$. O curso clínico da doença é quase sempre uma massa progressiva com metástase e morte de $70 \%$ em três anos a desde o diagnóstico ${ }^{5}$. Dick et al. ${ }^{12}$ apontam os pulmões, cérebro e osso, nesta ordem, como os locais mais comuns de metástase, enquanto outros apontam os ossos como os mais acometidos ${ }^{5,17}$. Linfadenopatia é evento atípico ${ }^{12}$.

Do ponto de vista otorrinolaringológico, os PNETs são extremamente raros. Ibarburen et al. ${ }^{5}$ revisaram 17 casos e apenas três deles tiveram origem na cabeça e pescoço enquanto nenhum teve como sítio primário a cavidade nasal ou seio paranasal. Em termos de sintomatologia, pode ocorrer perda auditiva e paralisia facial, devido acometimento dos VII e VIII nervos cranianos no ângulo pontocerebelar, por sua vez, consequência da disseminação de tumores centrais através da leptomeninge ${ }^{16}$. pPNET acometendo seios paranasais é extremamente incomum ${ }^{11}$. Alobid et al. ${ }^{11}$ descreveu um paciente de 23 anos com obstrução nasal unilateral, rinorréia e epistaxe recorrente, tendo sido identificada uma massa avermelhada à endoscopia nasal. Na tomografia computadorizada, a massa tinha origem no seio maxilar esquerdo e cavidade nasal, com extensão para outros seios paranasais e destruição óssea com invasão do assoalho da órbita esquerda e fossa pterigopalatina, cuja biópsia demonstrou tratar-se de pPNET.

O diagnóstico é complementado através de exames de imagem, principalmente a tomografia computadorizada (TC) e a ressonância nuclear magnética (RM). Em estudo para avaliar características dos tumores neuroectodérmicos, Dick et al. ${ }^{12}$ concluíram que as características mais típicas de tais tumores na TC foram: massa heterogênea com densidade de partes moles com captação após ad- 
ministração de contraste de forma irregular. Nenhum tumor de cabeça e pescoço apresentou calcificação à imagem. À ressonância magnética, os tumores foram isointensos ou levemente hiperintensos em T1 e em T2 pPNETs apresentaram-se com hipersinal ${ }^{12}$. Além disso, a TC é mais sensível para detecção de metástase pulmonar, enquanto a RM fornece mais informações sobre invasão de partes moles, sendo exames complementares ${ }^{12}$. Para Ibarburen et al. ${ }^{5}$ as características mais comuns na TC são uma massa bem definida, heterogênea, não calcificada, com áreas císticas e com captação de contraste. Na RM, as lesões foram isointensas em T1, hiperintensas em $\mathrm{T} 2^{4}$ e apresentaram realce (heterogêneo ou homogêneo) após administração de gadolíneo. TC e RM são essenciais no planejamento cirúrgico e na avaliação do grau de ressecabilidade do tumor (principalmente a RM) ${ }^{5}$.

A cintilografia óssea também deve ser realizada de rotina nos pacientes com diagnóstico de PNET, uma vez que a tomografia do sítio de origem não é capaz de demonstrar metástase óssea na maioria dos casos e esta é uma das mais comuns ${ }^{12}$. Esses pacientes têm pior prognóstico e podem beneficiar-se com quimioterapia mais intensa ${ }^{12}$.

O tratamento dos tumores neuroectodérmicos primitivos inclui, de uma forma geral, ressecção cirúrgica, irradiação e quimioterapia. A cirurgia é importante tanto para o diagnóstico patológico, já que estes tumores apresentam características radiológicas inespecíficas, quanto para redução do volume do tumor $^{16}$. Embora muitos autores advoguem a cirurgia como tratamento inicial de escolha, a quimioterapia neoadjuvante tem mostrado excelentes resultados na regressão do tumor, facilitando a ressecção e diminuindo o risco intraoperatório ${ }^{1}$. Mesmo que a quimioterapia alcance uma resposta completa, a cirurgia deve ser realizada para eliminar as células malignas no tecido cicatricial. Além disso, radioterapia pós operatória e quimioterapia também são preconizadas devido ao risco de recorrência local ou metástases a distância ${ }^{1}$. Em alguns pacientes é necessária uma segunda cirurgia para retirada de células malignas remanescentes (second look), principalmente nos que são submetidos primariamente à cirurgia ${ }^{1}$. Após completar o tratamento, os pacientes devem ter acompanhamento rigoroso em intervalos trimestrais nos primeiros dois anos e, após, seguimento semestral ${ }^{1}$.

A sobrevida dos pacientes portadores de pPNET é variável, apesar de o prognóstico ser considerado pobre ${ }^{18}$. Existe descrição de sobrevida de apenas 8 meses ${ }^{18}$, enquanto Khong et al. ${ }^{14}$ reportou sobrevida de $50 \%$ em 12 meses. A taxa geral de sobrevida em 5 anos em tumores localizados varia de $65 \%-74 \%$ e, para tumores metastáticos, varia de $25 \%-45 \%$, de acordo com Parham et al. ${ }^{19}$. Ibarburen et al. seguiram 17 pacientes, dos quais 9 morreram, sendo que 6 faleceram nos primeiros 8 meses $^{5}$. Algumas características são indicativas de mal prognóstico, como localização abdominal ou na região da cabeça e pescoço, bom como presença de metástase a distância ${ }^{12}$.

\section{CONCLUSÃO}

Os tumores neuroectodérmicos primitivos periféricos são entidades raras, principalmente na região da cabeça e pescoço. No entanto, devem fazer parte do diagnóstico diferencial dos tumores de partes moles - como linfoma, sarcoma de Ewing e rabdomiossarcoma - essencialmente em crianças e adolescentes. Devido ao grau de agressividade do tumor, o diagnóstico deve ser feito o mais precoce possível, através de história e exame clínicos detalhados, aliados a exames de imagem (TC, RM e cintilografia óssea), além da biópsia para confirmação histológica. O tratamento engloba quimioterapia, ressecção cirúrgica e radioterapia. Tendo conhecimento deste raro tipo de tumor, podemos garantir um diagnóstico mais rápido e acurado, aumentando, de maneira importante, a chance de sobrevida dos nossos pacientes.

\section{REFERÊNCIAS}

1. Zimmermann T, Blütters-Sawatzki R, Flechsenhar K, Padberg WM. Peripheral primitive neuroectodermal tumor: challenge for multimodal treatment. World $\mathrm{J}$ Surg. 2001;25:1367-72.

2. Santra G, Sinha PK, De D, Phaujdar S. Peripheral type of primitive neuroectodermal tumour arising from the left orbital floor. Singapore Med J. 2011;52(6):138-40.

3. Kampman WA, Kros JM, De Jong TH, Lequin MH. Primitive neuroectodermal tumours (PNETs) located

in the spinal canal; the relevance of classification as central or peripheral PNET: case report of a primary spinal PNET occurrence with a critical literature review. J Neurooncol. 2006;77:65-72.

4. Coffin CM, Dehner LP. Peripheral neurogenic tumors of the soft tissues in children and adolescents: a clinicopathologic study pf 139 cases. Pediatr Pathol. 1989;9:387.

5. Ibarburen C, Haberman JJ, Zerhouni EA. Peripheral 
primitive neuroectodermal tumors. CT and MRI evaluation. Eur J Radiol. 1996;21:225-32.

6. Kheinhues $P$, Bürger PC, Scheithauer BW. The new WHO classification of brain tumor. Brain Pathol. 1993;3:255.

7. Danner DB, Hruban RH, Pitt HA, Hayashi R, Griffin CA, Perlman EJ. Primitive neuroectodermal tumor arising in the pancreas. Mod Pathol. 1994;7:200.

8. Mor Y, Nass D, Raviv G, Neumann Y, Nativ O, Goldwasser B. Malignant peripheral neuroectodermal tumor (PNET) of the kidney. Med Pediatr Oncol. 1994;23:437.

9. Daya D, Lukka H, ClementPB. Primitive neuroectodermal tumor of the uterus: a report of four cases. Hum Pathol. 1992;23:1120.

10. Scherr GR, D’Ablaing G, Ouzounian JG. Peripheral primitive neuroectodermal tumor of the vulva. Gynecol Oncol. 1994;54:254.

11. Alobid I, Bernal-Sprekelsen M, Alós L, Benítez B, Traserra $\mathrm{J}$, Mullol J. Peripheral primitive neuroectodermal tumor of the left maxillary sinus. Acta Otolaryngol. 2003;123:776-8.

12. Dick EA, McHugh K, Kimber C, Michalski A. Imaging of non-central nervous system primitive neuroectodermal tumors: diagnostic features and correlation with outcome. Clin Radiol. 2001;56:206-15.

13. Khoury JD. Ewing sarcoma family of tumors. Adv Anat Pathol. 2005;12:212-20.

14. Khong PL, Chan GCF, Shek TWH, Tam PKH, Chan FL. Imaging of peripheral PNET: common and uncommon locations. Clin Radiol. 2002;57:272-7.

15. Thiele CJ. Biology of pediatric peripheral neuroectodermal tumors. Cancer Metastasis Rev. 1991;10:311-9.

16. Yan Y, Xu T, Chen J, Hu G, Lu Y. Intraespinal Ewing's sarcoma/primitive neuroectodermal tumors. J Clin Neurosci. 2011;18:601-6.

17. Askin FB, Rosai J, Sibley RK, Dehner LP, McAlister $\mathrm{WH}$. Malignant small cell tumor of the thoracopulmonary region in childhood. A distinctive clinicopathological entity of uncertain histogenesis. Cancer. 1979;43:243851.

18. Zhang W, Zhao L, Huang X, Cai PQ, Xu GX. Computed Tomography imaging of anterior and middle mediastinal Ewing sarcoma/primitive neuroectodermal tumors. Thorac Imaging. 2010;25:168-72.

19. Parham DM, Hijazi Y, Steinberg SM, Meyer WH, Horowitz M, Tzen CY, Wexler LH, Tsokos M. Neuroectodermal differentiation in Ewing's sarcoma family of tumors does not predict tumor behavior. Hum Pathol. 1999;30:911-8. 